\title{
A SET OF INDEPENDENT CONDITIONS THAT A REAL FUNCTION BE EVERYWHERE DIFFERENTIABLE†
}

\author{
BY F. W. PERKINS
}

1. Introduction. The purpose of this paper is to give a set of conditions which characterize those real functions of a real variable which are differentiable $\ddagger$ for all values of that variable. These conditions are somewhat analogous in spirit to the set of fundamental properties of definite integrals formulated by Lebesgue. $\S$

In $\$ 2$ we state the conditions, in $\$ 3$ we discuss their relation to differentiation, and in $\$ 4$ we prove that they are independent.

2. The Conditions. We consider a set $C$ of real functions of the real variable $x,(-\infty<x<\infty)$. We suppose that to each function $f(x)$ in $C$ there corresponds a real function $\bar{f}(x)$, $(-\infty<x<\infty)$, not necessarily in $C$. We impose on the set $C$ and on the correspondence between $f(x)$ and $\bar{f}(x)$ certain restrictions which are formulated as four conditions :\|

I. The function $X(x)=x$ is a function in $C$; moreover, there exist constants $x_{1}$ and $q$ such that $\bar{X}\left(x_{1}\right)=1$ and $\bar{X}(x)$ is different from $q$ for all $x$.

$\dagger$ This paper is a revision of a communication presented to the Society, December 27, 1934, under the title $A$ set of completely independent postulates for differentiation.

$\ddagger$ We consider a function differentiable for $x=x_{0}$ if and only if the difference quotient approaches a finite limit for $x=x_{0}$.

$\S \mathrm{H}$. Lebesgue, Leçons sur l'Intégration et la Recherche des Fonctions Primitives (Borel Collection), 1904, pp. 98, 99.

\|It will be noted that Condition I is compound in nature, inasmuch as it can be resolved into the following three propositions:

$\mathrm{I}^{\prime}$. The function $X(x)=x$ is in $C$.

$I^{\prime \prime}$. If the function $X(x)=x$ is in $C$, then there exists a constant $x_{1}$ such that $\bar{X}\left(x_{1}\right)=1$.

$I^{\prime \prime \prime}$. If the function $X(x)=x$ is in $C$, then there exists a constant $q$ such that $\bar{X}(x)$ is different from $q$ for all $x$.

Condition III is also compound. 
II. Given any constant $x_{2}$ and any function $f(x)$ in $C$, then $g(x)=f\left(x+x_{2}\right)$ is a function in $C$, and $\bar{g}(x)=\bar{f}\left(x+x_{2}\right)$.

III. There exists a constant $x_{3}$ such that if $f_{1}(x)$ and $f_{2}(x)$ are functions in $C$, and $k$ an arbitrary constant, then the function $F(x)=f_{1}(x)+k f_{2}(x)$ is a function in $C$, and $\bar{F}\left(x_{3}\right)=\bar{f}_{1}\left(x_{3}\right)+k \bar{f}_{2}\left(x_{3}\right)$.

IV. There exists a constant $x_{4}$ such that if $C$ contains functions $f_{1}(x)$ and $f_{2}(x)$ for which $f_{1}\left(x_{4}\right)=f_{2}\left(x_{4}\right)=\bar{f}_{1}\left(x_{4}\right)=0$, and $\bar{f}_{2}\left(x_{4}\right)>0$, then corresponding to any such pair of functions there is a neighborhood of $x_{4}$ throughout which $f_{1}(x) \leqq\left|f_{2}(x)\right|$.

3. Everywhere Differentiable Functions. This section is devoted to the demonstration of the following theorem.

TheOREM. A necessary and sufficient condition that a function $f(x)$ have a derivative for every value of $x$ is that $f(x)$ belong to some set $C$ for which conditions I-IV hold. If $f(x)$ belongs to any such set $C$, then the associated function $\bar{f}(x)$ is necessarily the derivative of $f(x)$.

In order to establish this theorem, we first prove three lemmas.

LEMMA 1. If $f_{1}(x)$ and $f_{2}(x)$ are any two functions in $C$, and if $a_{1}$ and $a_{2}$ are any constants, then $F^{*}(x)=a_{1} f_{1}(x)+a_{2} f_{2}(x)$ is a function in $C$, and $\bar{F}^{*}(x)=a_{1} \bar{f}_{1}(x)+a_{2} \bar{f}_{2}(x)$.

In III we may set $f_{1}(x)=f_{2}(x)=X(x)$, and so none of the first three postulates are vacuous. If we set $f_{1}(x)=f_{2}(x)=X(x)$ and $k=-1$, we see from III that the function $Z(x)=0$ is in $C$, and that $\bar{Z}\left(x_{3}\right)=0$. By now setting $f_{1}(x)=Z(x)$ we conclude that if $f(x)$ is any function in $C$, and $k$ any constant, then $F(x)=k f(x)$ is a function in $C$, and $\bar{F}\left(x_{3}\right)=k \bar{f}\left(x_{3}\right)$. Hence, under the hypotheses of this lemma, $a_{1} f_{1}(x), a_{2} f_{2}(x)$, and $F^{*}(x)$ are functions in $C$, and

$$
\bar{F}^{*}\left(x_{3}\right)=a_{1} \bar{f}_{1}\left(x_{3}\right)+a_{2} \bar{f}_{2}\left(x_{3}\right) .
$$

We shall now show that for an arbitrarily chosen constant $\xi$ we have

$$
\bar{F}^{*}(\xi)=a_{1} \bar{f}_{1}(\xi)+a_{2} \bar{f}_{2}(\xi) .
$$

From II, 


$$
g_{1}(x)=f_{1}\left(x+\xi-x_{3}\right), \quad g_{2}(x)=f_{2}\left(x+\xi-x_{3}\right),
$$

and

$$
g^{*}(x)=F^{*}\left(x+\xi-x_{3}\right)
$$

are functions in $C$. Since

$$
g^{*}(x)=a_{1} g_{1}(x)+a_{2} g_{2}(x),
$$

we infer from III that

$$
\bar{g}^{*}\left(x_{3}\right)=a_{1} \bar{g}_{1}\left(x_{3}\right)+a_{2} \bar{g}_{2}\left(x_{3}\right),
$$

whence

$$
\bar{F}^{*}(\xi)=a_{1} \bar{f}_{1}(\xi)+a_{2} \bar{f}_{2}(\xi) .
$$

This completes the proof of Lemma 1.

LEMMA 2. If $A(x)=a x+b$, where $a$ and $b$ are arbitrary constants, then $A(x)$ is in $C$, and $\bar{A}(x)=a$.

Consider the function $B(x)=b$. We may write

$$
B(x)=X(x+b)-X(x),
$$

and so from I, II, and III we see that $B(x)$ is in $C$. Since

$$
B\left(x+x_{2}\right)=B(x),
$$

it follows from II that

$$
\bar{B}\left(x+x_{2}\right)=\bar{B}(x),
$$

and so $\bar{B}(x)$ is a constant. In particular, the function $u(x)=1$ is in $C$, and $\bar{u}(x)$ is a constant, which we shall denote by $\bar{u}$. Now

$$
B(x)=b \cdot u(x)+0 \cdot u(x),
$$

and so, using Lemma 1,

$$
\bar{B}(x)=b \bar{u} .
$$

Consequently, using II and Lemma 1, we infer that

$$
\bar{X}(x+b)=\bar{X}(x)+b \bar{u} .
$$

Hence we see from a further application of $I$ that

$$
\bar{X}\left(x_{1}+b\right)=1+b \bar{u},
$$


for any constant $b$. Suppose, now, that $\bar{u} \neq 0$; then we may set $b=(q-1) / \bar{u}$, so as to have

$$
\bar{X}\left(x_{1}+\frac{q-1}{\bar{u}}\right)=q .
$$

This contradicts $I$, and so shows that $\bar{u}=0$. Consequently $\bar{X}\left(x_{1}+b\right)=1$, and since $b$ is an arbitrary constant this implies that $\bar{X}(x)=1$.

Now the function $A(x)$ given in the statement of this lemma may be expressed in the form

$$
A(x)=a X(x)+B(x) .
$$

Hence $A(x)$ is in $C$, and $\bar{A}(x)=a$, as was to be proved.

Lemma 3. Given any constant $\xi$, the set $C$ contains two functions $f_{1}(x)$ and $f_{2}(x)$ such that $f_{1}(\xi)=f_{2}(\xi)=\bar{f}_{1}(\xi)=0$, and $\bar{f}_{2}(\xi)>0$. Moreover, corresponding to any constant $\xi$ and a pair of functions $f_{1}(x)$ and $f_{2}(x)$ so related to it, there exists a neighborhood of $x=\xi$ throughout which $\left|f_{1}(x)\right| \leqq\left|f_{2}(x)\right|$.

Given any $\xi$, the functions $f_{1}(x)=0$ and $f_{2}(x)=x-\xi$ are in $C$ and satisfy the relations

$$
f_{1}(\xi)=f_{2}(\xi)=\bar{f}_{1}(\xi)=0, \text { and } \bar{f}_{2}(\xi)>0 .
$$

Hence the first part of this lemma is valid.

Suppose now that $f_{1}(x)$ and $f_{2}(x)$ are any functions for which these relations are satisfied, relatively to a preassigned $\xi$. Let

$$
g_{1}(x)=f_{1}\left(x-x_{4}+\xi\right) \text { and } g_{2}(x)=f_{2}\left(x-x_{4}+\xi\right),
$$

where $x_{4}$ is the constant the existence of which is asserted in IV. By virtue of II, the functions $g_{1}(x)$ and $g_{2}(x)$ are known to be in $C$; also

$$
\bar{g}_{1}(x)=\bar{f}_{1}\left(x-x_{4}+\xi\right) \text { and } \bar{g}_{2}(x)=\bar{f}_{2}\left(x-x_{4}+\xi\right) .
$$

Moreover,

$$
g_{1}(x)=g_{2}\left(x_{4}\right)=\bar{g}_{1}\left(x_{4}\right)=0, \text { and } \bar{g}_{2}\left(x_{4}\right)>0 .
$$

Hence, by IV, there exists a positive constant $\delta^{\prime}$ such that

$$
g_{1}\left(x_{4}\right) \leqq\left|g_{2}(x)\right|, \text { when }\left|x-x_{4}\right| \leqq \delta^{\prime},
$$


which is equivalent to saying that

$$
f_{1}(x) \leqq\left|f_{2}(x)\right| \text {, when }|x-\xi| \leqq \delta^{\prime} .
$$

Using Lemma 1 we see that we may replace $f_{1}(x)$ by the function $-f_{1}(x)$ in the present discussion. It follows that there exists a positive constant $\delta^{\prime \prime}$ such that

$$
-f_{1}(x) \leqq\left|f_{2}(x)\right| \text {, when }|x-\xi| \leqq \delta^{\prime \prime} .
$$

Hence

$$
\left|f_{1}(x)\right| \leqq\left|f_{2}(x)\right|, \text { when }|x-\xi| \leqq \delta,
$$

where $\delta$ is the smaller of $\delta^{\prime}$ and $\delta^{\prime \prime}$, or their common value if $\delta^{\prime}=\delta^{\prime \prime}$. Hence the validity of Lemma 3 is established.

We are now ready to prove the theorem stated at the beginning of this section. The necessity of the condition for differentiability there given is immediately obvious, for clearly I, II, III, and IV are each satisfied if $C$ is the set of all functions $f(x)$ which have a derivative $f^{\prime}(x)$ for every $x$, and if $\bar{f}(x)$ is identical with $f^{\prime}(x)$.

To complete the proof of the theorem we shall show that an arbitrarily chosen function $f(x)$ in $C$ has a derivative $f^{\prime}(x)$, and that $\bar{f}(\xi)=f^{\prime}(\xi)$, for an arbitrarily chosen constant $\xi$.

From Lemmas 1 and 2 we see that the function

$$
f_{1}(x)=f(x)-\{\bar{f}(\xi) x+[f(\xi)-\xi \bar{f}(\xi)]\}
$$

is in $C$, inasmuch as $f(x)$ is in $C$, and that

$$
\bar{f}_{1}(x)=\bar{f}(x)-\bar{f}(\xi) .
$$

We note that

$$
f_{1}(\xi)=\bar{f}_{1}(\xi)=0 .
$$

Consider also the function

$$
f_{2}(x)=\lambda x-\lambda \xi,
$$

where $\lambda$ is an arbitrary positive constant. Using Lemma 2, we see that $f_{2}(x)$ is in $C$; moreover,

$$
f_{2}(\xi)=0, \text { and } \bar{f}_{2}(\xi)=\lambda>0 .
$$

We now apply the second part of Lemma 3 to these functions 
$f_{1}(x)$ and $f_{2}(x)$. We infer that, for fixed $\xi$, to every positive $\lambda$ there corresponds a positive $\delta$ such that

$$
\left|f_{1}(x)\right| \leqq \lambda|x-\xi|,
$$

for values of $x$ such that $|x-\xi| \leqq \delta$. Since $f_{1}(\xi)=0$, we therefore have

$$
\left|\frac{f_{1}(\xi+\Delta x)-f_{1}(\xi)}{\Delta x}\right| \leqq \lambda, \text { when } 0<|\Delta x| \leqq \delta .
$$

Since $\lambda$ is any positive quantity, we infer that $f_{1}{ }^{\prime}(\xi)$ exists and that $f_{1}{ }^{\prime}(\xi)=0$. Recalling the definition of $f_{1}(x)$, we see that this implies that $f^{\prime}(\xi)$ exists and that $f^{\prime}(\xi)=\bar{f}(\xi)$. This completes the proof of the theorem.

4. The Independence of the Conditions. In order to establish the independence of the conditions I-IV, we must show that the set $C$ and the rule for determining $\bar{f}(x)$ can be so chosen that any given one of the conditions is invalid, and the remaining conditions valid.

In our first three examples we choose $C$ as the set of all functions $f(x)$ which are everywhere differentiable. If we set $\bar{f}(x)=2 f^{\prime}(x)$, then it is immediately obvious that $I$ is invalid and II, III, and IV are valid. If, however, we set $\bar{f}(x)=f^{\prime}(2 x)$, then II is invalid (as may be seen by choosing $x_{2}=1$ and $f(x)=x^{2}$ ), but each of the remaining postulates is valid. $\dagger$ If we set $\bar{f}(x)=\left|f^{\prime}(x)\right|$, then III is invalid (failing, for example, when $f_{1}(x)=x, f_{2}(x)=-x$ and $\left.k=1\right)$, but I, II, and IV are valid.

Finally, to obtain an example in which IV alone is invalid, we choose $C$ as the set of all real functions $f(x),(-\infty<x<\infty)$, and set $\bar{f}(x)=f(x+1)-f(x)$. That I, II, and III are valid can be established without difficulty. The invalidity of IV is immediately apparent from the generality of the set $C$.

Dartmouth College

$\dagger$ If we set $f(x)=X(x)=x$, then $f^{\prime}(x) \equiv 1$, or, replacing $x$ by $2 x, f^{\prime}(2 x) \equiv 1$, so that $\bar{X}(x) \equiv 1$. Hence I is valid. The validity of III and of IV (with $x_{4}=0$ ) is also easily established. 